\title{
Patterns in diet reveal foraging site fidelity of short-tailed shearwaters in the southeastern Bering Sea
}

\author{
Cheryl L. Baduini ${ }^{1,3, *}$, George L. Hunt Jr. ${ }^{1,4}$, Alexei I. Pinchuk ${ }^{2}$, Kenneth O. Coyle ${ }^{2}$ \\ ${ }^{1}$ Department of Ecology and Evolutionary Biology, University of California, Irvine, California 92697, USA \\ ${ }^{2}$ Institute of Marine Science, University of Alaska, Fairbanks, Alaska 99775, USA \\ ${ }^{3}$ Present address: Joint Science Department, The Claremont Colleges, Claremont, California 91711, USA \\ ${ }^{4}$ Present address: School of Aquatic and Fishery Sciences, Box 355020, University of Washington, Seattle, Washington 98195, USA
}

\begin{abstract}
The short-tailed shearwater Puffinus tenuirostris is an apex predator in the southeastern Bering Sea ecosystem. During 1997 to 1999, a period of great variability in the Bering Sea, we used a multi-pronged approach to study transfer of carbon and nitrogen to short-tailed shearwaters through analysis of stomach contents of birds collected while foraging, and stable isotope and fatty acid composition of tissues from shearwaters and their prey. Two conclusions result from these 3 analyses of feeding history. First, short-tailed shearwaters demonstrated localized differences in diet with respect to sampling location and season, indicating that shearwaters feed in discrete locations long enough (several weeks) to reflect regional differences in prey availability. Second, elevated $\delta^{15} \mathrm{~N}$ levels ( 1 to $2 \%$ ) in the liver of shearwaters in the fall of 1997 and 1998 were likely a response to nutritional stress and overturn of nutrients in the tissues of birds or elevated isotope levels in tissues of prey, rather than an increase in the trophic level of the diet. Over the 3 yr period, shearwater diets switched from adult euphausiids Thysanoessa raschii and T. inermis (usually females with spermatophores) taken in spring to an increase in the amount of fish (Pacific sandlance Ammodytes hexapterus), juvenile Gadidae fishes and larval walleye pollock Theragra chalcograma particularly in fall (1997 excepted). For each location and year, there was a consistent trend in isotopic values, with an increase of 3 to $5 \%$ for $\delta^{15} \mathrm{~N}$ and 1 to $3 \%$ for $\delta^{13} \mathrm{C}$ between shearwaters and their primary prey. The resolution of our sampling techniques indicates little movement of shearwater flocks among sample locations for periods of up to several weeks.
\end{abstract}

KEY WORDS: Short-tailed shearwater · Puffinus tenuirostris · Euphausiid · Gadid fishes · Stable isotope $\cdot$ Fatty acid $\cdot$ Diet $\cdot$ Prey

\section{INTRODUCTION}

During their annual occupation of the southeastern Bering Sea, short-tailed shearwaters Puffinus tenuirostris constitute the greatest biomass of seabirds in this ecosystem. Up to 16 million birds have been recorded from April to October (Schneider \& Shuntov 1993) with flocks reaching 10 s to 100 s of thousands of birds (Guzman 1981, Hunt et al. 1981, Gould et al. 1982). As consumers and producers of carbon and nitrogen, these great numbers of shearwaters can have a significant contribution to nutrient flow in this ecosystem during summer (Schneider \& Hunt 1982, Schneider et al. 1986). Thus, as an apex predator, the short-tailed shearwater is a good indicator species for observing trophic transfer of nutrients through the food web from lower to higher trophic levels and for detecting anomalous changes in ecosystem function.

As part of the Inner Front Study, during 1997 to 1999 we had the opportunity to examine the foraging eco- 
logy of short-tailed shearwaters (Napp \& Hunt 2001, Hunt et al. 1999, 2002a,b, Napp et al. 2002, Stabeno \& Hunt 2002, Jahncke et al. 2005). This comprehensive project incorporated 6 research cruises (2 seasons $\mathrm{yr}^{-1}$ ) covering more than $500 \mathrm{~km}^{2}$ of the southeastern Bering Sea and spanning both the warmest water period recorded (during an El Niño Southern Oscillation Event in 1997/1998), and an unusually cold period (during a La Niña Southern Oscillation event, in 1999). This project provided an opportunity to look at both spatial and temporal trends in nutrient flow to shorttailed shearwaters.

In this paper, a multi-pronged approach was employed to study the diet of short-tailed shearwaters. We quantified stomach contents of birds collected while foraging and analyzed the levels of enrichment of stable isotopes of carbon and nitrogen in the tissues of shearwaters and their prey. Additionally, a cursory sampling of fatty acids from the tissues of shearwaters and their prey was made. Our objective was to use these methods to detect temporal changes in diet using analyses with different time signatures. Diet analysis through examination of stomach contents would be indicative of most immediate diet, including the current meal taken while foraging. Stable isotope and fatty acid values, with turnover times on the order of days and weeks, would be indicative of diet incorporated over a longer time period. A second objective was to document spatial and temporal patterns in shearwater diets. We were particularly interested in determining whether diet changed significantly among years, seasons, or by sampling location. If shearwaters had discrete nutrient signatures at a particular location and season, then timing and location of sampling could be important factors in understanding transfer of nutrients to apex predators in this system.

This study occurred during an exceedingly warm year (1997) followed by another warm water year in 1998, and an anomalously cold year in 1999 (Stabeno \& Hunt 2002). In 1997, warm water (surface temperatures reached $14^{\circ} \mathrm{C}$ ) preceded a pervasive and persistent coccolithophore bloom, and up to $11 \%$ of the total short-tailed shearwater population observed died (live and dead birds counted), presumably of starvation (Baduini et al. 2001a), and diets shifted to smaller, lower-calorie prey (Hunt et al. 2002b). In 1998, the entire water column was warm $\left(6\right.$ to $\left.12^{\circ} \mathrm{C}\right)$ and shearwaters had low body lipid content $(<1$ to $9 \%$ of wet body mass), yet no major die-off was observed (Baduini et al. 2001b). In 1999, surface waters were cooler $\left(8\right.$ to $10^{\circ} \mathrm{C}$ ) than the long-term average, and shearwater body lipid content was significantly greater than in fall 1997 or 1998 (mean = 10\% of wet body mass). A preliminary examination of stomach contents of shearwaters collected during the study is presented in Hunt et al. (2002b).

Documentation of stomach contents is a traditional method for assessing the diets of seabirds. Stomach contents provide a record of the current or last meal eaten by a predator prior to sampling. Unless sampled regularly over an extended period, stomach contents reflect short-term diet and can be biased by the more rapid digestion of soft-bodied prey (i.e. crustaceans; Gaston \& Nettleship 1981) and by the retention of hard body parts (squid beaks and otoliths; Wilson et al. 1985). However, when sampled frequently and in abundance, they provide useful information on changes in diet over time and space. In our study, we collected an extensive set of stomach contents sampled in 2 seasons, over $3 \mathrm{yr}$, and totaling 264 individuals with prey contents. This dataset provided a useful basis for the study of trophic transfer and changes to short-tailed shearwaters.

More recently, in the 1990s, stable isotope analysis has come into use as a tool for studying trophic relationships between predators and their prey, particularly with seabirds (Hobson 1990, 1991, 1993, Hobson \& Montevecchi 1991, Rau et al. 1992). This approach is based on the premise that stable isotopes of carbon $\left({ }^{13} \mathrm{C} /{ }^{12} \mathrm{C}\right)$ and particularly nitrogen $\left({ }^{15} \mathrm{~N} /{ }^{14} \mathrm{~N}\right)$ show a step-wise enrichment with trophic level in marine ecosystems. The average difference in ${ }^{13} \mathrm{C} /{ }^{12} \mathrm{C}$ (hereafter defined $\left.\delta^{13} \mathrm{C}\right)$ and ${ }^{15} \mathrm{~N} /{ }^{14} \mathrm{~N}\left(\delta^{15} \mathrm{~N}\right)$ between a consumer and its prey in marine food webs is $\pm 1 \%$ and $\pm 3.2 \%$, respectively (Michener \& Schell 1994). Thus an increase of $1 \%$ in $\delta^{13} \mathrm{C}$ and $3.2 \%$ in $\delta^{15} \mathrm{~N}$ in the tissues of a predator over time may be indicative of a change in diet by 1 trophic level. It has also been shown experimentally that $\delta^{15} \mathrm{~N}$ but not $\delta^{13} \mathrm{C}$ values can be elevated by 1 to $2 \%$ in avian tissues when individuals have been fasting or are nutritionally stressed (Hobson et al. 1993, Cherel et al. 2005a). We used this relationship to assess the presence of nutritional stress in shearwaters during the warm water periods when other studies had shown that body lipids were low and birds were emaciated (Baduini et al. 2001b).

Fatty acid signatures have also been used in the examination of diet and feeding ecology of apex predators (Iverson 1993, Iverson et al. 1995, 1997, Raclot et al. 1998, Dahl et al. 2003, Connan et al. 2005). Fatty acids are components of lipid tissue that comprise triglycerides and wax esters. Similar to stable isotope analysis, this method is based upon the principle that the pattern of fatty acid composition in the predator's prey will be reflected in the tissues of the predator, although the composition of fatty acid make-up can change as predators digest, absorb, and reassemble components of prey tissue (Iverson et al. 2004). Fatty acid signatures are thought to permit a 
more fine-scale examination of diet than stable isotopes because fatty acid composition is significantly different not only among trophic levels, but among species and life-stages of a species (Iverson et al. 2002). Thus, lipid tissue can be sampled as a means of determining the species composition of prey that has been assimilated by a predator over a period of days to weeks.

Both stable isotope and fatty acid analyses can provide information about changes in diet over time and space, but the diet can be best interpreted through a comparison of these methods with signatures in prey. We also believe that knowledge about diet from stomach contents is essential for confirming changes in diet in combination with these 2 other indirect methods. Together, these 3 methods provide a more complete view of trophic transfer of nutrients than any 1 method alone. For this reason, we used our extensive sample base of stomach contents analyses and stable isotope data, coupled with a less extensive sampling of fatty acids, to understand spatial and temporal changes in prey use by short-tailed shearwaters in the southeastern Bering Sea ecosystem.

\section{MATERIALS AND METHODS}

Sampling program. The project incorporated 6 research cruises to the southeastern Bering Sea between May 1997 and August 1999. Two research cruises were conducted each year. The first, in late May and June (spring) coincided with the arrival of short-tailed shearwaters, and occurred during or shortly after the spring phytoplankton bloom. The second, from the end of July to September (late summer or fall), occurred as the shearwaters completed molt and began to put on fat prior to their migration to breeding grounds in southeastern Australia. Cruise dates were 27 May to 28 June, 1997; 27 August to 12 September, 1997; 23 May to 24 June, 1998; 15 August to 7 September 1998; 17 May to 19 June 1999; and 19 July to 22 August 1999. Sampling on these cruises was concentrated in 4 grids that also received extensive oceanographic sampling (Fig. 1; Jahncke et al. 2005). Slime Bank and Port Moller grids were along the north side of the Alaska Peninsula, the Cape Newenham grid covered a large area from the shore off Cape Newenham to the $70 \mathrm{~m}$ isobath, and the Nunivak Island grid covered a long narrow area from close inshore off Nunivak Island southwest about $150 \mathrm{~km}$. Thus, this project provided an opportunity to look at both spatial and temporal patterns in nutrient flow to short-tailed shearwaters.

Stomach content analyses. Short-tailed shearwaters were collected under permit. Although there were some individuals from which more than 1 type of sample was taken, in some cases samples for analysis of stomach contents, stable isotopes, fatty acids, and total body lipids were obtained from different individuals collected at a given place and time (Table 1). All individuals collected were examined for stomach contents

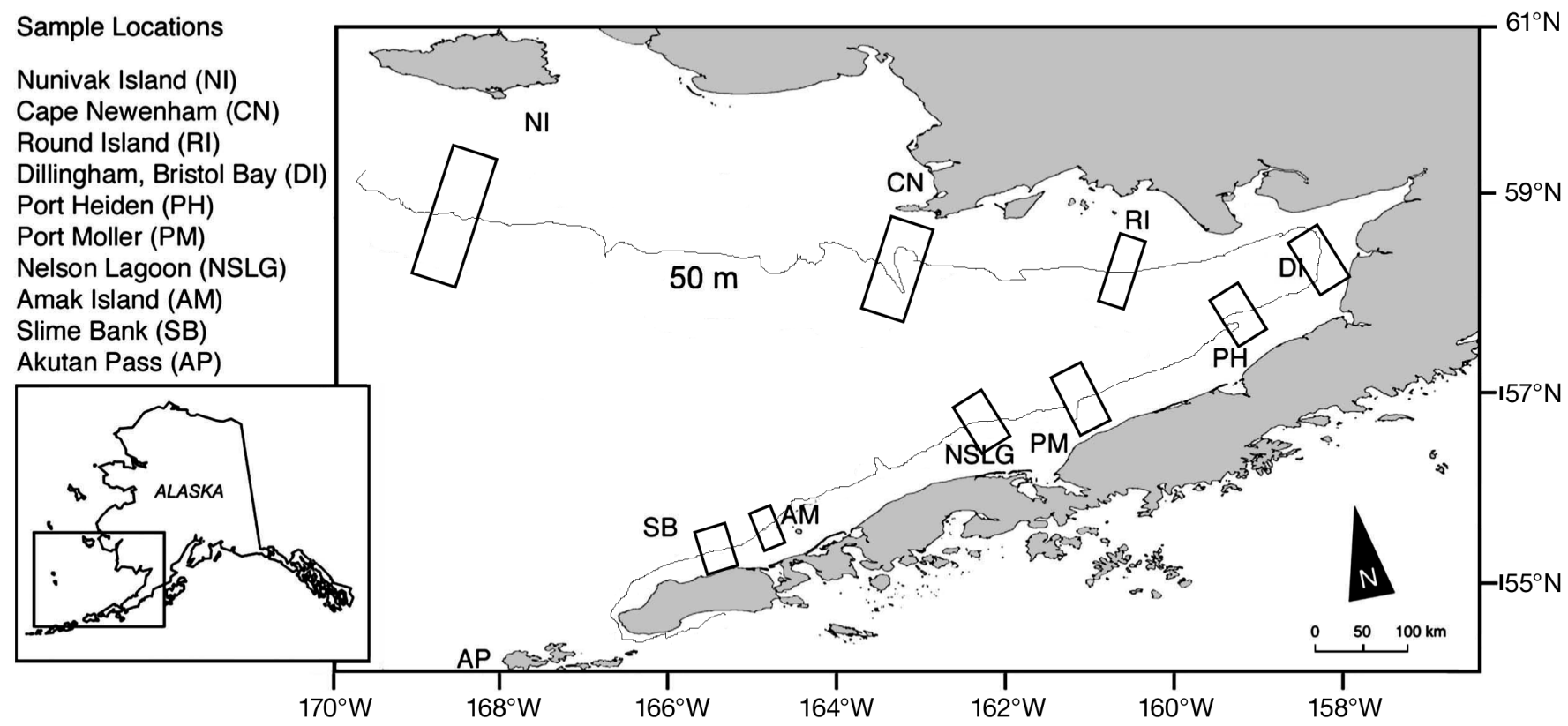

Fig. 1. Study site and sampling locations for short-tailed shearwaters in the southeastern Bering Sea, 1997 to 1999 . Squares indicate stations within the Nunivak Island, Cape Newenham, Round Island, Bristol Bay, Port Heiden, Port Moller, Nelson Lagoon, Amak Island, Slime Bank and Akutan Pass grids. The inner shelf domain is inside of the $50 \mathrm{~m}$ isobath, the middle domain between the 50 and $100 \mathrm{~m}$ isobaths 
and these samples represent the most complete sampling of individuals. Samples collected from birds at a given location and season were considered nonindependent and were averaged.

Diet composition was determined by collecting short-tailed shearwaters while foraging or sitting on the water within each sampling grid (Fig. 1). Approximately 3 to 10 birds per flock were sampled in each grid, usually when feeding, to be certain that the birds had obtained their prey near where they were collected. Upon collection, proventriculus and gizzard contents were removed, weighed, and preserved separately in $80 \%$ ethanol. Only proventriculus content information is reported here. Wet weight of alcoholpreserved proventriculus contents, displacement volume, and direct counts of each individual stomach were used to determine the diet of birds. Euphausiids were identified to species, sex (although not used in Index of Relative Importance, IRI calculation), and life stage (adult vs. juvenile). Crab larvae, usually megalopa and some zoea stages, were combined for counts. We did not calculate IRI values for amphipods and mysids, which were observed in less than $10 \%$ of all samples. Other invertebrate zooplankton and fish consumed were identified to the lowest possible taxon.

Based upon number, volume, and frequency of occurrence of prey items, the IRI was calculated for each study location within each season following Gould et al. (2000) and Pinkas et al. (1971):

$$
\mathrm{IRI}=\% \mathrm{~F}(\% \mathrm{~N}+\% \mathrm{~V})
$$

where $\mathrm{F}=$ frequency of occurrence, $\mathrm{N}=$ number of items within a sample, and $\mathrm{V}=$ volume of items counted. Percent frequency of occurrence was calculated by taking the number of individual stomachs containing a particular prey item divided by the total number of stomachs sampled containing prey in the sampled location $(\times 100)$. Percent number was calculated by taking the total number of individuals sampled for a specific taxon divided by the total number of all prey items counted in all stomachs of birds sampled at a particular location $(\times 100$; i.e. Slime Bank, spring 1997). Percent volume is the total volume of an individual prey item (ml) summed over all specimens in a given sampling divided by the total summed volumes of all prey $(\times 100)$. To determine percent volume in cases where prey items of dif- ferent taxa (i.e. different species of euphausiids) were identified but grouped in one total volume, the numerical percent of each prey species in a proventriculus was determined and multiplied by total volume (to calculate $\%$ volume). Percent IRI of a particular prey item is the IRI of a single taxon divided by the IRI of the total diet (sum of all IRIs) for a particular location and season.

Stable isotope analyses. We collected 1489 samples for stable isotope analysis from short-tailed shearwaters $(\mathrm{N}=241$; Table 1$)$ and their potential prey $(\mathrm{N}=$ 1248; Table 2). Invertebrate and prey samples were collected from net hauls using the MOCNESS (Multiple Opening-Closing Net Sampling System). Stable isotopes of shearwaters were sampled by drying approximately 5 to $10 \mathrm{~g}$ of liver in an oven at $60^{\circ} \mathrm{C}$ for $24 \mathrm{hr}$. Dried tissue samples were pulverized into powder and prepared for analysis in a mass spectrometer. All zooplankton samples were acidified to remove carbonates. Two subsamples per tissue were analyzed for isotopic ratios.

Isotope samples were analyzed in the lab of Dr. Donald Schell (University of Alaska). $\delta^{15} \mathrm{~N}$ and $\delta^{13} \mathrm{C}$ values were measured using Elemental AnalysisIsotope Ratio Mass Spectrometry (EA-IRMS), Delta+ XL system. This method utilizes a Costech Elemental

Table 1. Puffinus tenuirostris. Number of short-tailed shearwaters sampled for stomach contents, stable isotopes, and fatty acid analyses during the 1997 to 1999 Bering Sea Inner Front Study. Although there is some overlap of individuals sampled for all 3 analyses, birds sampled for one technique (stomach contents) are not necessarily the same individuals sampled for another technique (fatty acids)

\begin{tabular}{|c|c|c|c|c|c|c|c|}
\hline Season & Location & $\begin{array}{l}\text { Stomach } \\
\text { contents }\end{array}$ & $\begin{array}{c}\text { Stable } \\
\text { isotopes }\end{array}$ & $\begin{array}{l}\text { Fatty } \\
\text { acids }\end{array}$ & $\begin{array}{c}\text { Ger } \\
\text { Female }\end{array}$ & $\begin{array}{c}\text { nder }(\% \\
\text { Male }\end{array}$ & $\begin{array}{l}\% \mathrm{~N}) \\
\text { Unknown }\end{array}$ \\
\hline \multirow{3}{*}{$\begin{array}{c}\text { Spring } \\
1997\end{array}$} & Slime Bank & 23 & 8 & 23 & 68.2 & 18.2 & 13.6 \\
\hline & Port Moller & 7 & 0 & 3 & 57.14 & 42.86 & 0 \\
\hline & Cape Newenham & $\mathrm{m} 12$ & 10 & 3 & 66.7 & 33.3 & 0 \\
\hline \multirow{2}{*}{$\begin{array}{l}\text { Fall } \\
1997\end{array}$} & Slime Bank & 32 & 22 & 22 & 28 & 69 & 0 \\
\hline & Nunivak Island & 14 & 20 & 22 & 35.3 & 58.8 & 3.1 \\
\hline \multirow{4}{*}{$\begin{array}{c}\text { Spring } \\
1998\end{array}$} & Slime Bank & 7 & 10 & 0 & 50 & 50 & 0 \\
\hline & Port Moller & 18 & 19 & 0 & 36.8 & 57.9 & 5.3 \\
\hline & Dillingham Bay & 19 & 20 & 0 & 50 & 45 & 5 \\
\hline & Cape Newenham & m 7 & 7 & 0 & 43 & 57 & 0 \\
\hline \multirow{3}{*}{$\begin{array}{l}\text { Fall } \\
1998\end{array}$} & Slime Bank & 10 & 10 & 3 & 0 & 90 & 10 \\
\hline & Cape Newenham & n 26 & 26 & 8 & 38.5 & 61.5 & 0 \\
\hline & Nunivak Island & 4 & 5 & 0 & 100 & 0 & 0 \\
\hline \multirow{6}{*}{$\begin{array}{c}\text { Spring } \\
1999\end{array}$} & Slime Bank & 19 & 20 & 8 & 45 & 45 & 10 \\
\hline & Port Moller & 18 & 10 & 4 & 53.3 & 13.3 & 33 \\
\hline & Port Heiden & 6 & 4 & 0 & 50 & 33.3 & 17 \\
\hline & Nelson Lagoon & 9 & 10 & 3 & 29.4 & 23.5 & 47 \\
\hline & Round Island & 5 & 6 & 0 & 42.9 & 28.6 & 43.3 \\
\hline & Cape Newenham & 5 & 5 & 3 & 40 & 20 & 40 \\
\hline \multirow{4}{*}{$\begin{array}{c}\text { Summer } \\
1999\end{array}$} & Slime Bank & 8 & 9 & 0 & 62.5 & 37.5 & 0 \\
\hline & Port Moller & 7 & 8 & 0 & 71.4 & 28.5 & 0 \\
\hline & Cape Newenham & 8 & 12 & 0 & 50 & 33.3 & 17 \\
\hline & Nunivak Island & 13 & 14 & 0 & 46.2 & 30.8 & 23 \\
\hline
\end{tabular}




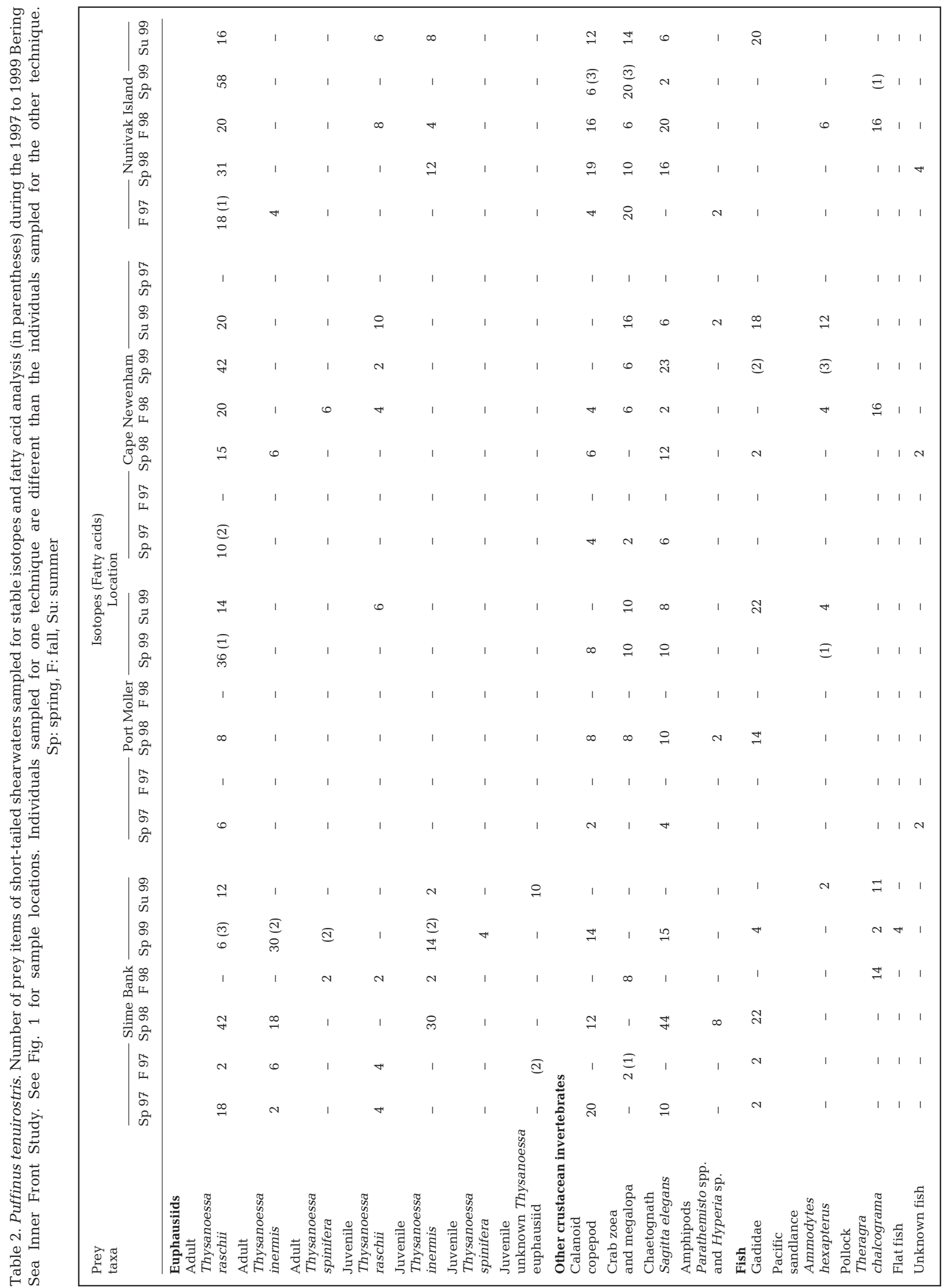


Analyzer (ESC 4010), and a Finnigan MAT Conflo III interface with a Delta+XL Mass Spectrometer. Samples run prior to 1999 (1997 and 1998 samples) were run on a Europa 20-20 Continuous Flow Mass Spectrometer interfaced with a Roboprep Elemental Analyzer. Dried, homogenous samples were placed in tin capsules and placed in the EA autosampler. Samples were dropped into the EA where they were combusted. The $\mathrm{N}_{2}$ and $\mathrm{CO}_{2}$ combustion gases were separated chromatographically and then transferred to the IRMS, where the isotopes were measured. $\delta^{15} N_{\text {air }}$ and $\delta^{13} C_{\mathrm{PDB}}$ values are reported in reference to international isotope standards.

Stable isotope concentrations were expressed in delta notation as parts per thousand according to the following:

$$
\delta \mathrm{X}=\left[\left(\mathrm{R}_{\text {sample }} / \mathrm{R}_{\text {standard }}\right)-1\right] \times 1000
$$

where $\mathrm{X}$ is ${ }^{15} \mathrm{~N}$ or ${ }^{13} \mathrm{C}$ and $\mathrm{R}$ is the corresponding ratio ${ }^{15} \mathrm{~N} /{ }^{14} \mathrm{~N}$ and ${ }^{13} \mathrm{C} /{ }^{12} \mathrm{C}$. $\mathrm{R}_{\text {standard }}$ for ${ }^{15} \mathrm{~N}$ and ${ }^{13} \mathrm{C}$ are the atmospheric $\mathrm{N}_{2}$ (AIR) and Pee Dee Belemnite (PDB) standard, respectively. An analysis of total $\mathrm{C} / \mathrm{N}$ ratios in bird and zooplankton tissues demonstrated that lipids did not comprise a significant portion of the sample (all but $4 \mathrm{C} / \mathrm{N}$ values were less than 4.0 and ranged from 1.2 to 3.5; McConnaughey \& McRoy 1979), and thus did not influence the outcome of isotopic ratio results. Thus, we did not lipid normalize our delta isotope ratios.

Fatty acid signature analyses. Shearwaters and their prey were also sampled for fatty acid composition (short-tailed shearwaters $\mathrm{N}=102$, Table 1 ; and prey $\mathrm{N}=35$; Table 2). Due to sampling constraints, some locations and years were missing from our dataset and we restrict our discussion to samples collected in 1997 (Table 1). Duplicate samples of sub-cutaneous fatty tissue were collected from individual birds from below the neck area and stored in liquid nitrogen at sea until they were transferred to the laboratory and stored at $-20^{\circ} \mathrm{C}$ before analysis. Samples were analyzed for total lipid composition, which includes both polar and neutral lipids, in the laboratory of S. Iverson (Dalhousie University) using the modified Folch method (Budge et al. 2002, Iverson et al. 2004). Adipose tissue was collected from birds in 1997 that had little energy reserves, and thus the fatty acid composition of total lipids probably was influenced by the presence of structural lipids. Each sample was homogenized and $1.5 \mathrm{~g}$ tissue aliquots were extracted using 2:1 chloroform:methanol. Fatty acids were prepared and analyzed using a Perkin-Elmer Autosystem II capillary gas chromatograph (GC) with a flame ionization detector (ID) using a flexible fused silica column. Fatty acids are reported using shorthand nomenclature as follows: $A: B n-X$, where $A$ represents the number of carbon atoms, $B$ the number of double bonds, and $X$ the position of the double bond closest to the terminal methyl group. Each fatty acid was reported as weight percent of total fatty acids.

Differences among locations and seasons within a given year were analyzed using a multivariate Discriminate Function Analysis (STATISTICA 6.0) using the 17 fatty acids which had the largest overall variance and overall mean composition of $\geq 0.5 \%$ mass total fatty acids. A second criteria used for choosing which particular fatty acids to include in the analyses of shearwater diet was to include any fatty acid that had been shown to be informative as a dietary indicator in other studies of marine predators and their feeding habits (Iverson et al. 1997, 2002, 2004). Sixteen of the 17 (94\% similarity) fatty acids that we used in our analyses were determined informative in a study of the lipids of forage fish and invertebrates in Prince William Sound, Alaska (Iverson et al. 2002; Table 2). The exception was 22:5n-3, which, despite its large mean percent composition in shearwater tissues and prey, was not used in our analyses because it may be an intermediate fatty acid between 20:5n-3 and 22:6n-3 (Ackman et al. 1988; following methods in Iverson et al. 2002). Percentage values for fatty acids were transformed into log ratios prior to discriminant analyses by first renormalizing the values for the 17 fatty acids over $100 \%$. Since the log of 0 cannot be taken, 0 values $(n=$ 2 ) were changed to $0.005 \%$ prior to the calculation of the log ratio (following Iverson et al. 2002). A value of $0.005 \%$ was selected because it is below what is considered to be the minimum detectable level of a fatty acid $(0.01 \%)$ but is not so small as to result in extreme outliers following transformation (Iverson et al. 2002).

\section{RESULTS}

\section{Spatial and temporal heterogeneity in diet}

Stomach contents (IRI), stable isotope, and fatty acid analyses revealed feeding localization and specialization among seasons and years (Figs. 2 to 5). The principal prey items taken by short-tailed shearwaters in the southeastern Bering Sea during 1997 to 1999 were adult euphausiids, Thysanoessa raschii and T. inermis, juvenile Thysanoessa euphausiids, Pacific sandlance Ammodytes hexapterus, juvenile Gadidae fishes and age-0 pollock Theragra chalcograma (Fig. 2). Over the $3 \mathrm{yr}$ period, we observed a switch in the diet from adult euphausiids (usually $T$. raschii females with spermatophores) taken in spring (typically $>95 \%$ euphausiid IRI) to an increase in the amount of fish taken in fall (1997 excepted; 70 to $100 \%$ fish IRI, Fig 2.). There was also an increase in the amount of fish taken over the 


\begin{tabular}{|c|c|c|c|c|c|c|c|c|c|c|c|c|c|c|c|c|c|}
\hline 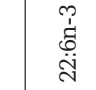 & 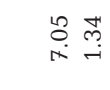 & 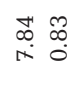 & $\begin{array}{l}\stackrel{8}{0} \overrightarrow{0} \\
\dot{i}\end{array}$ & 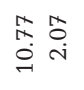 & 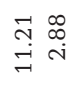 & $\begin{array}{l}\text { N } \\
\text { తi h } \\
\text { in }\end{array}$ & 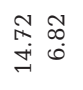 & 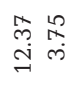 & 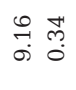 & 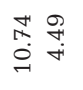 & $\underset{\infty}{m} \stackrel{m}{\rightarrow}$ & & 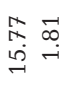 & 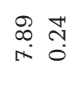 & 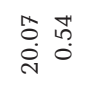 & సี. & 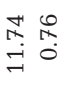 \\
\hline $\begin{array}{l}\text { 永 } \\
\text { À }\end{array}$ & 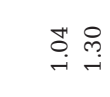 & \begin{tabular}{ll}
0 \\
$\stackrel{h}{h}$ \\
\hdashline
\end{tabular} & 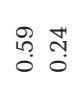 & 品 & $\begin{array}{ll}0 & 0 \\
\infty \\
0 \\
0\end{array}$ & $\begin{array}{cc}\overrightarrow{0} & \vec{m} \\
0 \\
0\end{array}$ & 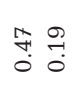 & 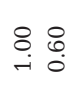 & $\begin{array}{l}\vec{A} \\
\rightarrow \\
\rightarrow\end{array}$ & 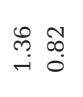 & 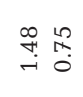 & & 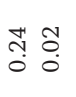 & $\stackrel{\infty}{\stackrel{0}{0}} \stackrel{0}{+}$ & $\begin{array}{l}8 \\
: \\
0 \\
0\end{array}$ & $\stackrel{n}{0}$ & $\begin{array}{ll}\infty & -1 \\
0 & 0 \\
0 & 0\end{array}$ \\
\hline 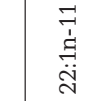 & 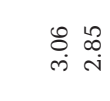 & $\stackrel{8}{8} \underset{i}{\stackrel{4}{4}}$ & 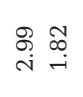 & 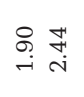 & 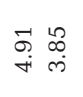 & 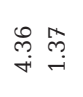 & $\stackrel{\text { 乌ి }}{i} \stackrel{\infty}{\rightarrow}$ & 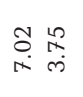 & 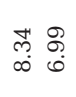 & 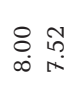 & 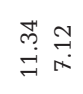 & & 市: & $\begin{array}{ll}\infty & 8 \\
\substack{\infty \\
0} & 0 \\
0 & 0\end{array}$ & $\stackrel{n}{0}: \stackrel{8}{0}$ & 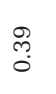 & $\begin{array}{l}\text { 菅: } \\
0\end{array}$ \\
\hline 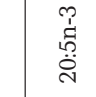 & 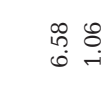 & 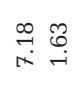 & ن & S̊ & $\begin{array}{l}\stackrel{0}{0} \\
\dot{b} \\
\dot{i}\end{array}$ & $\begin{array}{ll}\infty \\
\stackrel{\infty}{\infty} \\
\stackrel{\infty}{+} \stackrel{\leftrightarrow}{-}\end{array}$ & 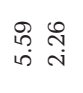 & 号 & 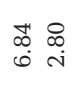 & 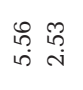 & 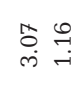 & & 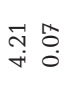 & 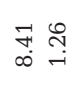 & 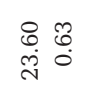 & 范 & 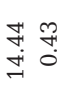 \\
\hline $\begin{array}{l}\text { i } \\
\stackrel{5}{7} \\
\stackrel{\text { iे }}{1}\end{array}$ & 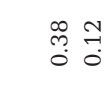 & 管 & ్ల & $\stackrel{\substack{\infty \\
\hdashline}}{\substack{0 \\
\hdashline}}$ & 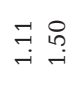 & \begin{tabular}{l} 
स. \\
\multirow{0}{*}{} \\
0
\end{tabular} & ్ㅜㅇ స్ & 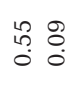 & 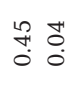 & 茯 & 府 & & సે & 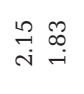 & $\underset{+i}{8} \stackrel{\infty}{0}$ & $\stackrel{ }{\stackrel{\rightarrow}{\rightarrow}}$ & $\begin{array}{ll}\overrightarrow{0} & \text { to } \\
0 & 0\end{array}$ \\
\hline 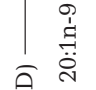 & 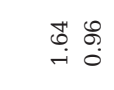 & $\stackrel{m}{\rightarrow} \underset{\sim}{\stackrel{\leftrightarrow}{-}}$ & $\stackrel{\vec{n}}{\sim} \stackrel{m}{\sim}$ & $\stackrel{\mathscr{\leftrightarrow}}{\leftrightarrow} \vec{i}$ & 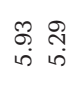 & 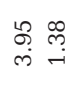 & 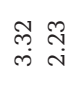 & în & 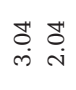 & 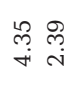 & 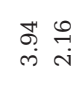 & & 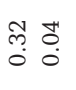 & 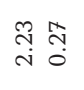 & 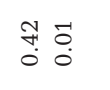 & $\underset{\leftrightarrow}{\mathscr{r}}$ & $\stackrel{\circ}{\because} \stackrel{0}{\circ}$ \\
\hline 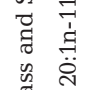 & 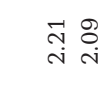 & $\stackrel{\infty}{\rightarrow} \underset{\rightarrow}{\stackrel{F}{\rightarrow}}$ & 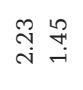 & 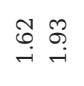 & 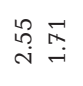 & 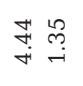 & 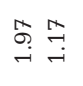 & 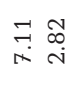 & 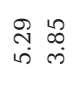 & 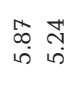 & 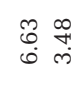 & & $\begin{array}{l}0 \\
\stackrel{0}{0} \\
0 \\
0\end{array}$ & $\stackrel{\leftrightarrow}{\rightarrow} \stackrel{\leftrightarrow}{\sigma}$ & $\begin{array}{ll}\mathscr{0} & -1 \\
0 & 0\end{array}$ & $\underset{0}{\vec{H}}$ & 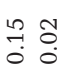 \\
\hline & $\begin{array}{ccc}\vec{x} & \infty \\
0 & 0 \\
0\end{array}$ & 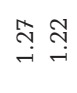 & 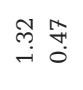 & 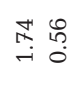 & 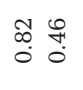 & $\stackrel{m}{\rightarrow}=$ & $\begin{array}{ll}0 & 0 \\
0 & 0 \\
0 & 0 \\
0\end{array}$ & $\underset{0}{-0} \stackrel{0}{0}$ & 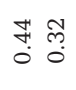 & N & 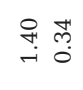 & & $\begin{array}{ll}D & 8 \\
0 & 8 \\
0 & 0\end{array}$ & $\underset{\oplus}{\stackrel{m}{\rightarrow}} \overrightarrow{0}$ & 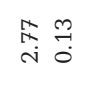 & $\stackrel{\text { N }}{\rightarrow}$ & 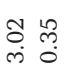 \\
\hline & 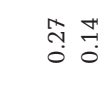 & 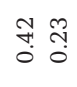 & 告 : & 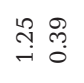 & 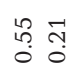 & 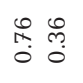 & 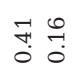 & 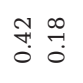 & $\begin{array}{ll}\substack{\text { N̦ } \\
0} \\
0\end{array}$ & 号 & : & & 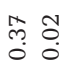 & 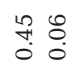 & 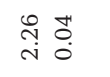 & $\vec{\sigma}$ & 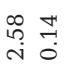 \\
\hline 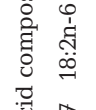 & 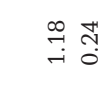 & $\stackrel{\infty}{\rightarrow} \underset{\sim}{\infty} \stackrel{\infty}{0}$ & 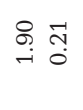 & 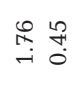 & 菅 & 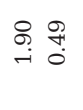 & 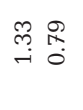 & 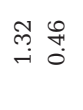 & 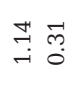 & 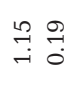 & $\vec{F}$ & & $\begin{array}{l}\text { क् } \\
i \\
i\end{array}$ & ने & 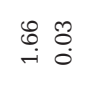 & : & \begin{tabular}{ll}
$\overrightarrow{0}$ & \multirow{1}{*}{} \\
$\stackrel{1}{0}$ & 0
\end{tabular} \\
\hline 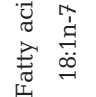 & $\begin{array}{l}\overrightarrow{0} . \\
0 \\
0\end{array}$ & 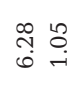 & 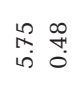 & $\stackrel{\leftrightarrow}{\stackrel{\leftrightarrow}{*}}$ & 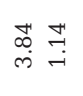 & 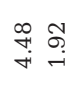 & $\begin{array}{l}\underset{+}{H} \underset{+}{\infty} \\
\stackrel{\sim}{\infty}\end{array}$ & 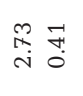 & 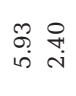 & 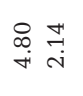 & 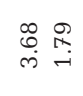 & & $\begin{array}{lll}\infty & 0 \\
\infty & 0 & 0 \\
\infty & \infty & 0 \\
0\end{array}$ & $\stackrel{0}{\infty} \stackrel{0}{\infty}$ & 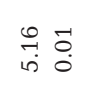 & 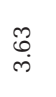 & $\begin{array}{ll}\vec{x} & ? \\
0 & 9 \\
0\end{array}$ \\
\hline 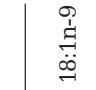 & 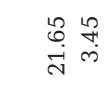 & 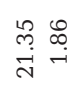 & 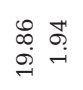 & 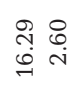 & 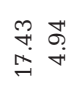 & 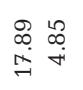 & 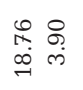 & $\begin{array}{l}\vec{n} \vec{\sigma} \\
\stackrel{\Delta}{\sim}\end{array}$ & $\begin{array}{l}\vec{X} \\
\underset{H}{\sim} \\
\vec{H}\end{array}$ & 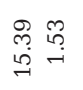 & 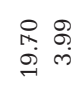 & & 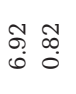 & 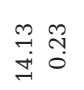 & $\begin{array}{rl}-\overrightarrow{0} & \infty \\
0 & 0 \\
0\end{array}$ & $\begin{array}{l}\vec{\infty} \\
\stackrel{\infty}{\infty} \\
i\end{array}$ & $\begin{array}{lll}\infty & 0 \\
\infty & 0 \\
0 & 0 & 0 \\
0\end{array}$ \\
\hline$\stackrel{\stackrel{0}{\oplus}}{\stackrel{0}{\rightarrow}}$ & 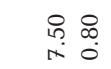 & 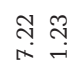 & 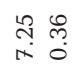 & 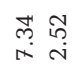 & 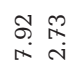 & 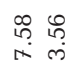 & $\begin{array}{l}\stackrel{\infty}{\sim} \stackrel{\infty}{\vec{H}} \\
\sigma\end{array}$ & $\begin{array}{ll}\overrightarrow{6} \\
\infty \\
\infty\end{array}$ & $\underset{0}{0} \stackrel{\infty}{\stackrel{\infty}{-}}$ & 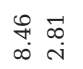 & તું & & 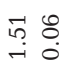 & 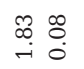 & $\underset{\sim}{\stackrel{0}{-}} \stackrel{0}{0}$ & 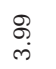 & 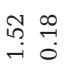 \\
\hline 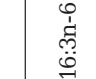 & $\begin{array}{l}\text { în } \\
0 \\
0\end{array}$ & 융웅 & 윰 & \begin{tabular}{l}
$\infty$ \\
$\stackrel{\infty}{0}$ \\
\hdashline
\end{tabular} & 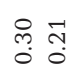 & $\stackrel{\substack{m \\
0}}{\stackrel{9}{0}}$ & 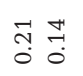 & 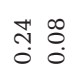 & 等: & 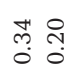 & ঙ্ণে & & $\begin{array}{l}\vec{X} \\
0 \\
0 \\
0\end{array}$ & 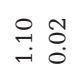 & 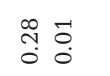 & $\stackrel{7}{0}$ & $\begin{array}{l}\text { 芯 } \\
0 \\
0\end{array}$ \\
\hline 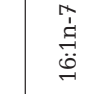 & 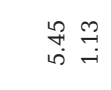 & $\begin{array}{l}\text { 芯 } \\
\dot{0}\end{array}$ & 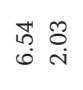 & $\begin{array}{l}: \\
\stackrel{\infty}{\infty} \stackrel{\infty}{0}\end{array}$ & $\begin{array}{ll}\mathscr{\infty} \\
\stackrel{n}{\infty} \stackrel{\leftrightarrow}{\sim}\end{array}$ & $\begin{array}{ll}\vec{N} & \stackrel{\infty}{\rightarrow} \\
\dot{m} & \stackrel{\sim}{i}\end{array}$ & 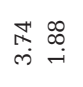 & 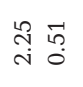 & $\begin{array}{l}\ddot{G} \\
\dot{i}\end{array} \vec{i}$ & 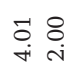 & 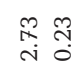 & & 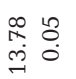 & 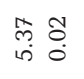 & $\begin{array}{l}\infty \\
\stackrel{\leftrightarrow}{i}\end{array}$ & $\underset{\infty}{\stackrel{\Delta}{\infty}}$ & 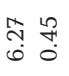 \\
\hline$\stackrel{\stackrel{\leftrightarrow}{\Theta}}{ }$ & 获 & 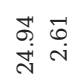 & 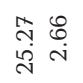 & 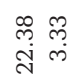 & 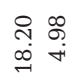 & 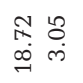 & 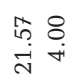 & 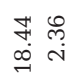 & 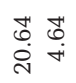 & $\begin{array}{l}\mathcal{O} \\
\stackrel{\leftrightarrow}{\sim} \\
\stackrel{\sim}{\sim}\end{array}$ & 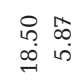 & & 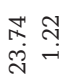 & 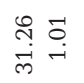 & 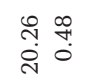 & $\begin{array}{l}\infty \\
\stackrel{\infty}{\rightarrow} \\
\dot{\rightarrow}\end{array}$ & 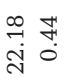 \\
\hline$\stackrel{\circ}{\stackrel{\longrightarrow}{+}}$ & $\begin{array}{l}\infty \\
\stackrel{\infty}{0} \stackrel{0}{0}\end{array}$ & 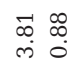 & 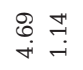 & \begin{tabular}{ll}
$\delta$ & 8 \\
& \multicolumn{1}{c}{}
\end{tabular} & 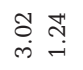 & 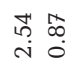 & 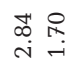 & 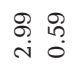 & 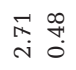 & $\begin{array}{l}\vec{N} 8 \\
\text { i }\end{array}$ & 学 & & 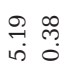 & $\stackrel{\overbrace{}}{\stackrel{m}{\infty}} \stackrel{\infty}{:}$ & $\stackrel{\infty}{\infty} \stackrel{\infty}{\rightarrow}$ & 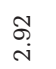 & 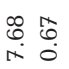 \\
\hline $\mathrm{z}$ & ๙ & $m$ & $m$ & ส & ส & $m$ & $\infty$ & $\infty$ & $m$ & $\nabla$ & $m$ & & N & & N & - & $m$ \\
\hline 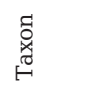 & $\begin{array}{l}\text { 岕 } \\
\text { 点 }\end{array}$ & $\begin{array}{l}\text { 䓌 } \\
\text { 点 }\end{array}$ & $\begin{array}{l}\text { 芴 } \\
\text { 点 }\end{array}$ & $\begin{array}{l}\text { 芴 } \\
\text { 点 }\end{array}$ & 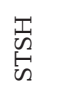 & $\begin{array}{l}\text { 䓌 } \\
\stackrel{5}{n}\end{array}$ & $\begin{array}{l}\text { 壳 } \\
\text { 点 }\end{array}$ & 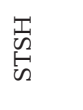 & $\begin{array}{l}\text { 臺 } \\
\text { 点 }\end{array}$ & $\begin{array}{l}\text { 岕 } \\
\text { 点 } \\
\omega\end{array}$ & $\begin{array}{l}\text { 䓌 } \\
\text { 点 }\end{array}$ & & 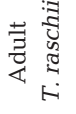 & 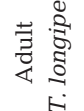 & 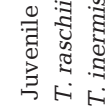 & 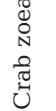 & 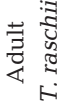 \\
\hline 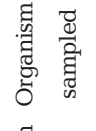 & 韋 & 莡 & 㺃 & : & : & 莡 & 密 & : & : & 密 & 㺃 & & 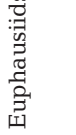 & 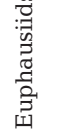 & 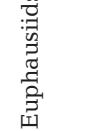 & 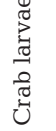 & 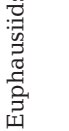 \\
\hline & 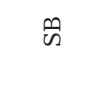 & $\sum_{a}$ & Z & 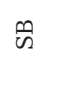 & z & 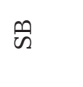 & Z & $\ddot{n}$ & $\begin{array}{l}0 \\
\text { W } \\
\text { Z }\end{array}$ & $\sum_{\Omega}$ & 乙 & & Z & u & $\ddot{n}$ & $\stackrel{\infty}{n}$ & Z \\
\hline 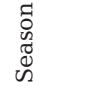 & 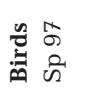 & $\begin{array}{l}\text { s. } \\
\text { के }\end{array}$ & $\begin{array}{l}\text { के } \\
\text { के }\end{array}$ & 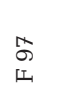 & $\begin{array}{l}\text { to } \\
\text { के }\end{array}$ & $\begin{array}{l}\infty \\
\infty \\
1\end{array}$ & 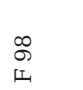 & $\begin{array}{l}8 \\
\text { के } \\
\text { की }\end{array}$ & $\begin{array}{l}8 \\
\text { \&े } \\
\text { ڤn }\end{array}$ & $\begin{array}{l}8 \\
\text { \&े } \\
\text { ஸे }\end{array}$ & $\begin{array}{l}8 \\
\text { Dे } \\
\text { in }\end{array}$ & 总 & $\begin{array}{l}\text { के } \\
\text { के }\end{array}$ & $\begin{array}{l}\text { के } \\
\text { on } \\
\text { की }\end{array}$ & 等 & 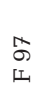 & 8 \\
\hline
\end{tabular}



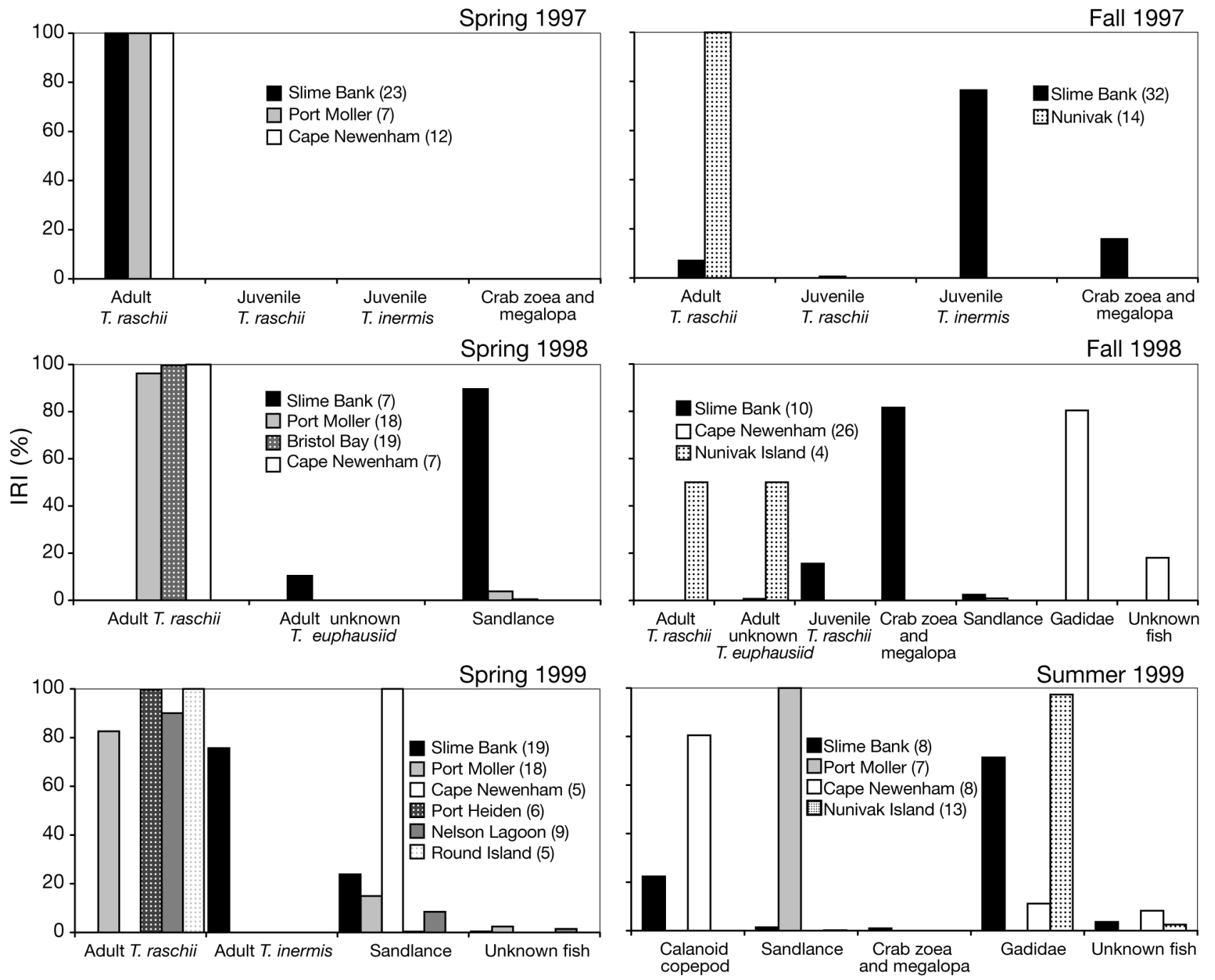

Fig. 2. Puffinus tenuirostris. Percent Index of Relative Importance (IRI) values for prey items of short-tailed shearwaters sampled among all study grids in 1997 to 1999. Sample sizes of stomach contents at each location are listed in parentheses. T.: Thysanoessa

3 yr period from 1997 to 1999, from <1\% IRI of fish in the diet in 1997 to $100 \%$ IRI of fish at Cape Newenham in spring 1999 and at Port Moller and Nunivak Island in summer 1999 (Fig. 2). The use of crab zoea and megalopa in fall 1997 and 1998, and Calanus marshallae copepods in summer 1999, also occurred.

With respect to sampling location, prey use included adult Thysanoessa raschii in most regions in spring 1997, 1998 and 1999. However, there were differences among locations in the IRI of other prey species. In the summer and fall samples, there was considerable variation in the IRIs of the different prey taxa at the locations sampled (Fig. 2). Similar to 1997, in fall 1998, prey use at Slime Bank included adult and juvenile Thysanoessa, as well as crab zoea and megalopa and some sandlance. In contrast, during fall 1998, juvenile gadids and age- 0 pollock were the most important prey items at Cape Newenham. Adult Thysanoessa euphausiids were taken by short-tailed shearwaters at Nunivak Island in fall 1998.

Short-tailed shearwaters $(\mathrm{N}=241)$ and their prey ( $\mathrm{N}=1248)$ displayed distinct isotopic values with respect to year, season and sample location (Figs. 3 to 7 ). A series of 1-way Kruskall-Wallis tests indicated significant differences among locations within a season in the $\delta^{13} \mathrm{C}$ and $\delta^{15} \mathrm{~N}$ values in the liver tissue of shearwaters (all $\mathrm{p}<0.001$; Fig. 7). Birds and prey sampled in a particular location within a season and year did not have overlapping values (or confidence intervals) with others collected elsewhere (except in spring 1999), indicating spatial and temporal heterogeneity.

Overall, there was an increasing trend in isotope values among trophic levels in the southeastern Bering Sea ecosystem from juvenile euphausiids to adult euphausiids, juvenile Gadidae fishes, sandlance, pollock, amphipods, and chaetognaths, with the highest 
values found in the liver tissue of short-tailed shearwaters (Figs. 3 to 6). For each location and year, there was a consistent trend with an increase in 3 to $5 \%$ o for $\delta^{15} \mathrm{~N}$ and 1 to $3 \%$ for $\delta^{13} \mathrm{C}$ between shearwaters and their primary prey (Figs. 3 to 6 ).

Our sampling of shearwater lipids and their prey in 1997 revealed seasonal and regional differences in fatty acid signatures (Fig. 8). There was a significant relationship between shearwaters and their prey for a particular season. In 1997, the first and second discriminant functions accounted for significant differences in fatty acid composition of short-tailed shearwaters among several locations sampled (Wilke's $\lambda<0.001$, $F_{85,304}=8.52, \mathrm{p}<0.001$; Fig. 8). All birds and prey sampled at locations in spring and fall (1997) were significantly different (all p < 0.05) except Slime Bank, Port Moller, and Cape Newenham in spring 1997. In the
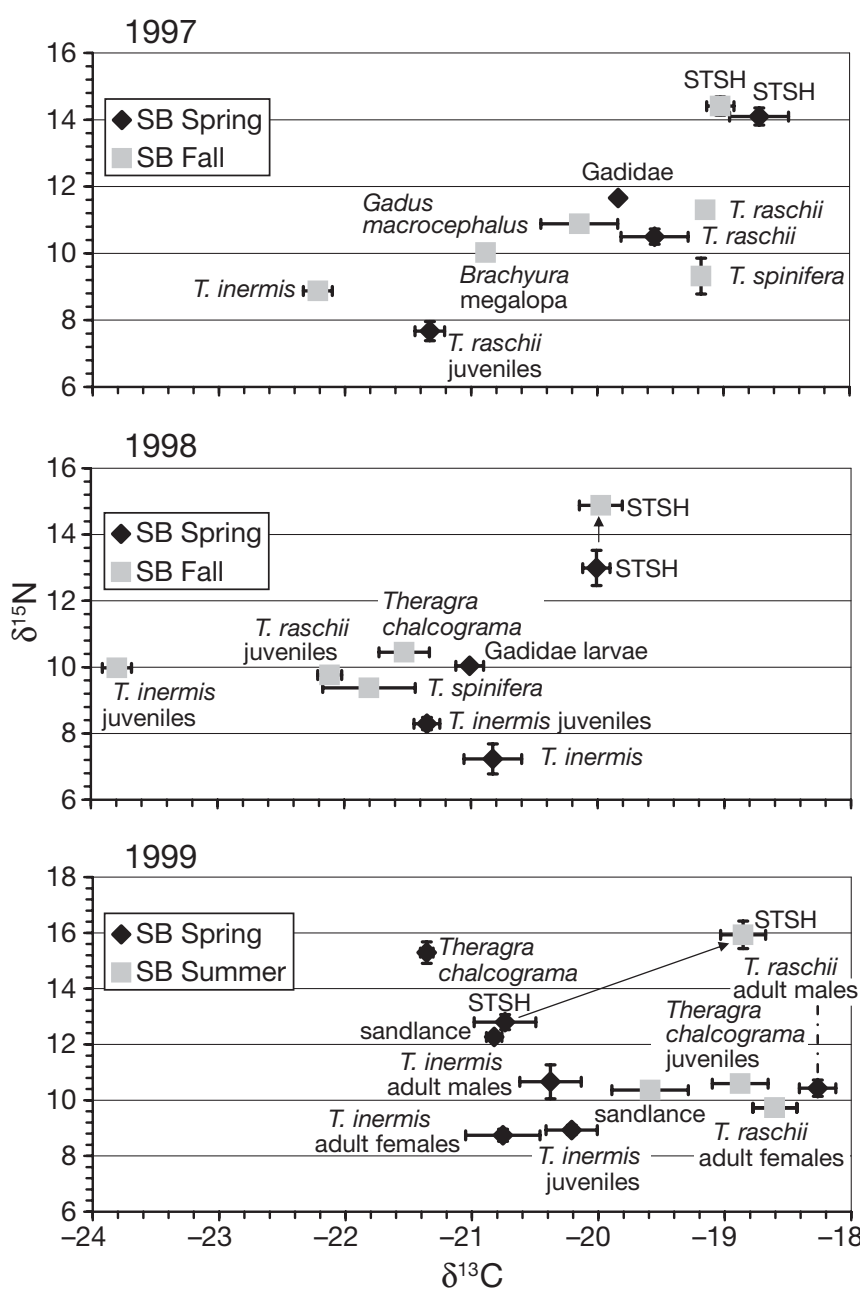

Fig. 3. Puffinus tenuirostris. Stable isotope values, $\delta^{15} \mathrm{~N}$ and $\delta^{13} \mathrm{C}$ for short-tailed shearwater (STSH) tissues (liver) and their prey by year and season off Slime Bank (SB) in the southeastern Bering Sea. Taxa labels are listed next to their corresponding mean \pm SE. Sample sizes are listed in Tables 1 \& 2. T.: Thysanoessa analysis of fatty acid patterns birds and prey are clustered together in spring and fall 1997 (Fig. 8).

A total of 17 (of 71 observed) fatty acids fitted our criteria for use in the analysis (Table 2) and they accounted for 86 to $95 \%$ of the mass of total fatty acids measured. The fatty acids, 16:0 and 18:4n-3, known to be important isomers in euphausiids, were abundant in the tissues of Thysanoessa sp. euphausiids and short-tailed shearwaters feeding on them. Likewise, the fatty acids 18:1n-9 (pollock), 22:6n-3 (sandlance tissue; Iverson et al. 2002), and 20:5n-3 (young pollock; Iverson et al. 1997) were abundant in the tissues of sandlance and pollock prey that we sampled and shearwaters that fed on them (Table 2).

\section{Diet, body condition and isotope changes}

We observed greater $\delta^{15} \mathrm{~N}$ values in the liver tissue of short-tailed shearwaters sampled at Slime Bank during fall 1997, when birds were emaciated and had lower total body lipid values compared to spring 1997 (Fig. 9; $t$-test between spring and fall 1997, Slime Bank, $t_{\text {two-tailed } 39}=1.746, \mathrm{p}=0.089$, mean difference $=0.9 \%$ o). Short-tailed shearwaters had $\delta^{15} \mathrm{~N}$ values that were elevated by an average $1.3 \%$ in fall compared to spring 1997 (Slime Bank), despite feeding on lower

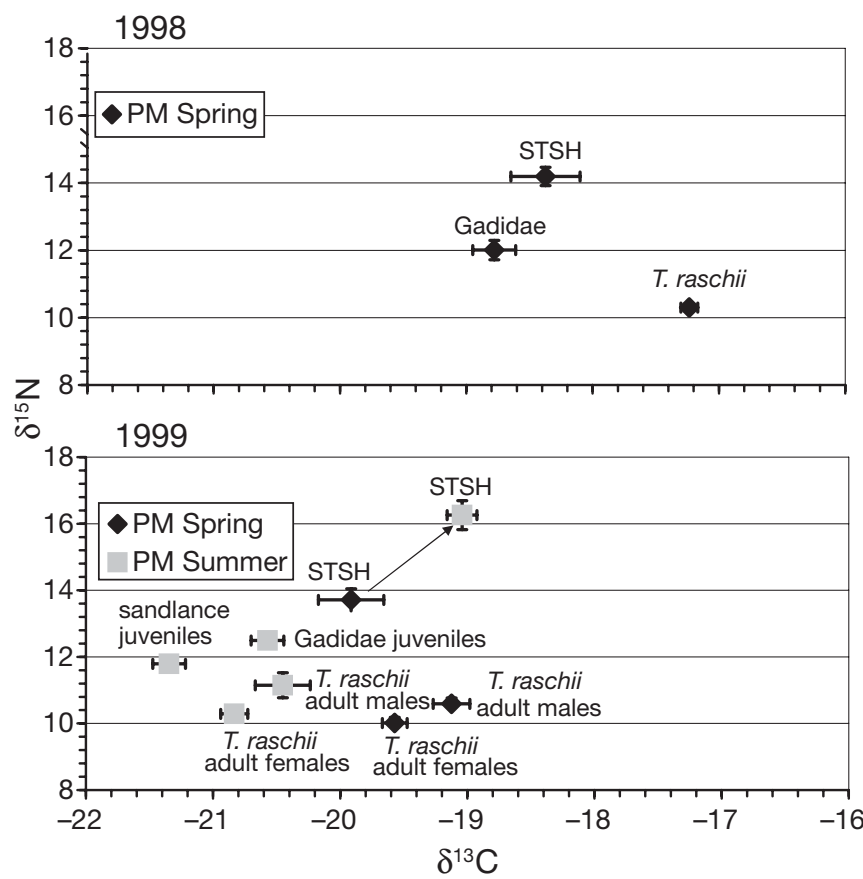

Fig. 4. Puffinus tenuirostris. Stable isotope values, $\delta^{15} \mathrm{~N}$ and $\delta^{13} \mathrm{C}$ for short-tailed shearwater (STSH) tissues (liver) and their prey by year and season off Port Moller (PM) in the southeastern Bering Sea. Taxa labels are listed next to their corresponding mean \pm SE. Sample sizes are listed in Tables 1 \& 2. T.: Thysanoessa 


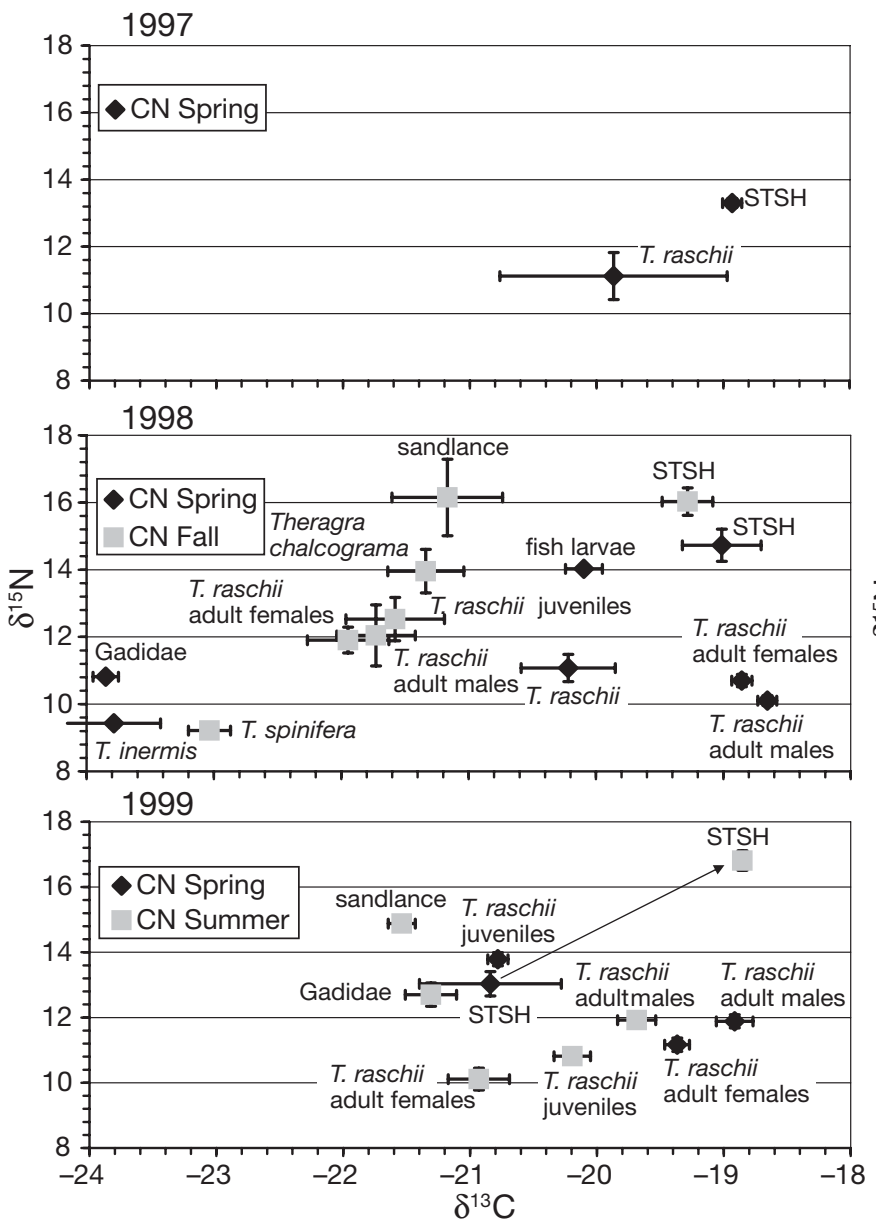

Fig. 5. Puffinus tenuirostris. Stable isotope values, $\delta^{15} \mathrm{~N}$ and $\delta^{13} \mathrm{C}$ for short-tailed shearwater (STSH) tissues (liver) and their prey by year and season off Cape Newenham (CN) in the southeastern Bering Sea. Taxa labels are listed next to their corresponding mean \pm SE. Sample sizes are listed in Tables 1 \& 2. T.: Thysanoessa

trophic levels in the fall. Similarly in $1998, \delta^{15} \mathrm{~N}$ values increased by 1.5 to $2 \%$ from spring to fall at Slime Bank and Cape Newenham (Fig. 9). Conversely, there was no significant increase in $\delta^{13} \mathrm{C}$ values observed at any location during this time. The greater $\delta^{15} \mathrm{~N}$ values observed in fall 1998 at Slime Bank occurred despite evidence from stomach contents that the shearwaters there were taking lower-trophic-level prey than in the spring. The isotope data indicate that prey tissues increased in $\delta^{15} \mathrm{~N}$ but not $\delta^{13} \mathrm{C}$ during this time.

In 1999, we observed increased $\delta^{15} \mathrm{~N}$ and $\delta^{13} \mathrm{C}$ values in the tissues of short-tailed shearwaters along with increased percent body lipid content and body condition compared to that observed for spring (Fig. 9). At all locations in 1999, shearwaters were feeding mainly on sandlance and adult euphausiids in spring and other types of fish in fall. This increase from spring to fall in 1999 was probably related to a shift in feeding at higher trophic levels.

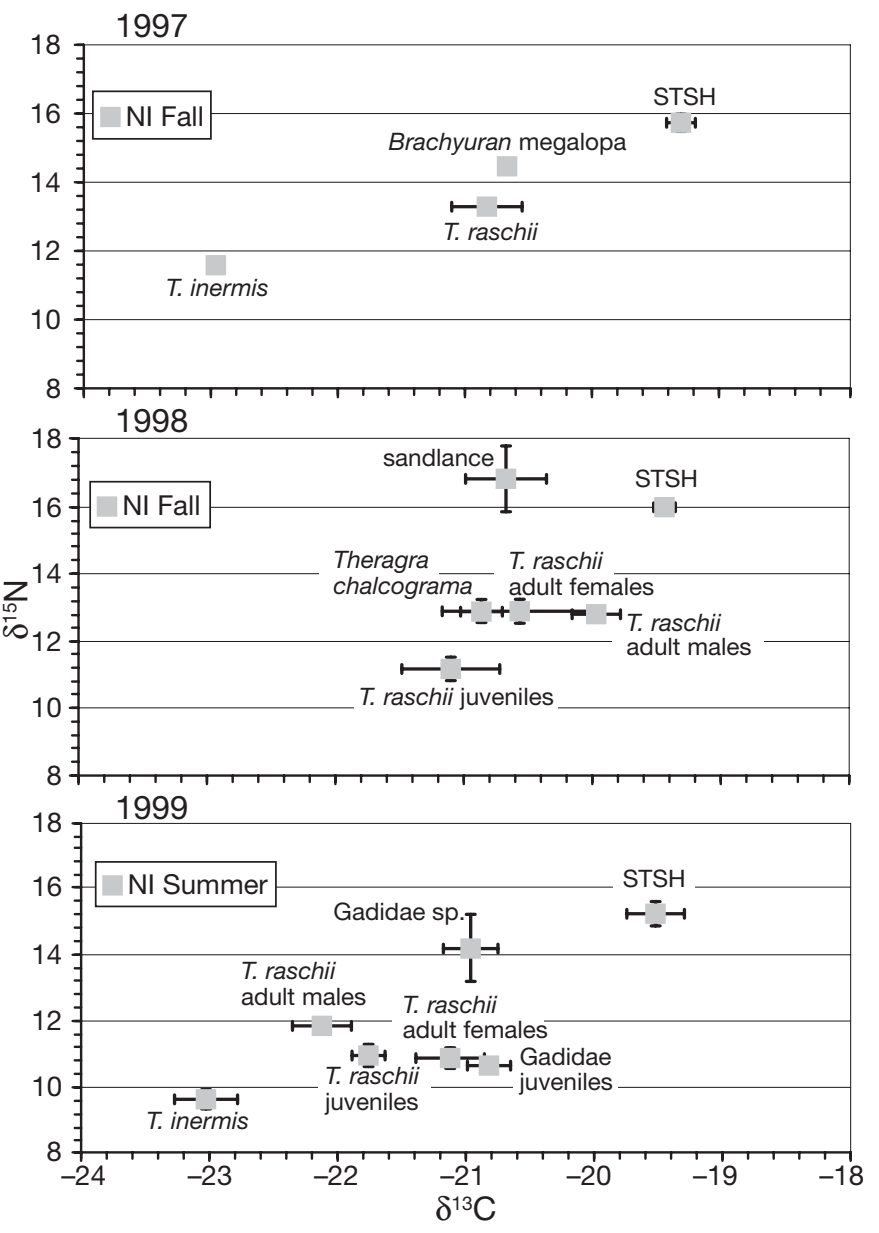

Fig. 6. Puffinus tenuirostris. Stable isotope values, $\delta^{15} \mathrm{~N}$ and $\delta^{13} \mathrm{C}$ for short-tailed shearwater (STSH) tissues (liver) and their prey by year and season off Nunivak Island (NI) in the southeastern Bering Sea. Taxa labels are listed next to their corresponding mean \pm SE. Sample sizes are listed in Tables 1 \& 2. T.: Thysanoessa

\section{DISCUSSION}

Two new observations resulted from the 3 analyses of feeding history of short-tailed shearwaters in the southeastern Bering Sea. First, we observed spatial and temporal heterogeneity in the diet among years, seasons, and sampling locations, indicating that shearwaters feed in discrete locations long enough (several weeks) to reflect regional differences in prey availability. Second, elevated $\delta^{15} \mathrm{~N}$ levels (1 to $2 \%$ ) in the liver of shearwaters in fall 1997 and 1998 were related to nutritional stress and overturn of nutrients in the tissues of birds, or were due to elevated levels of $\delta^{15} \mathrm{~N}$ in the prey items that birds were feeding upon.

In fall 1998, we found the greatest disparity in types of prey items taken with respect to sample locations, with juvenile euphausiids and crab zoea taken at Slime Bank, adult euphausiids taken at Nunivak Island, and Gadid fishes (especially age-0 pollock) taken at 


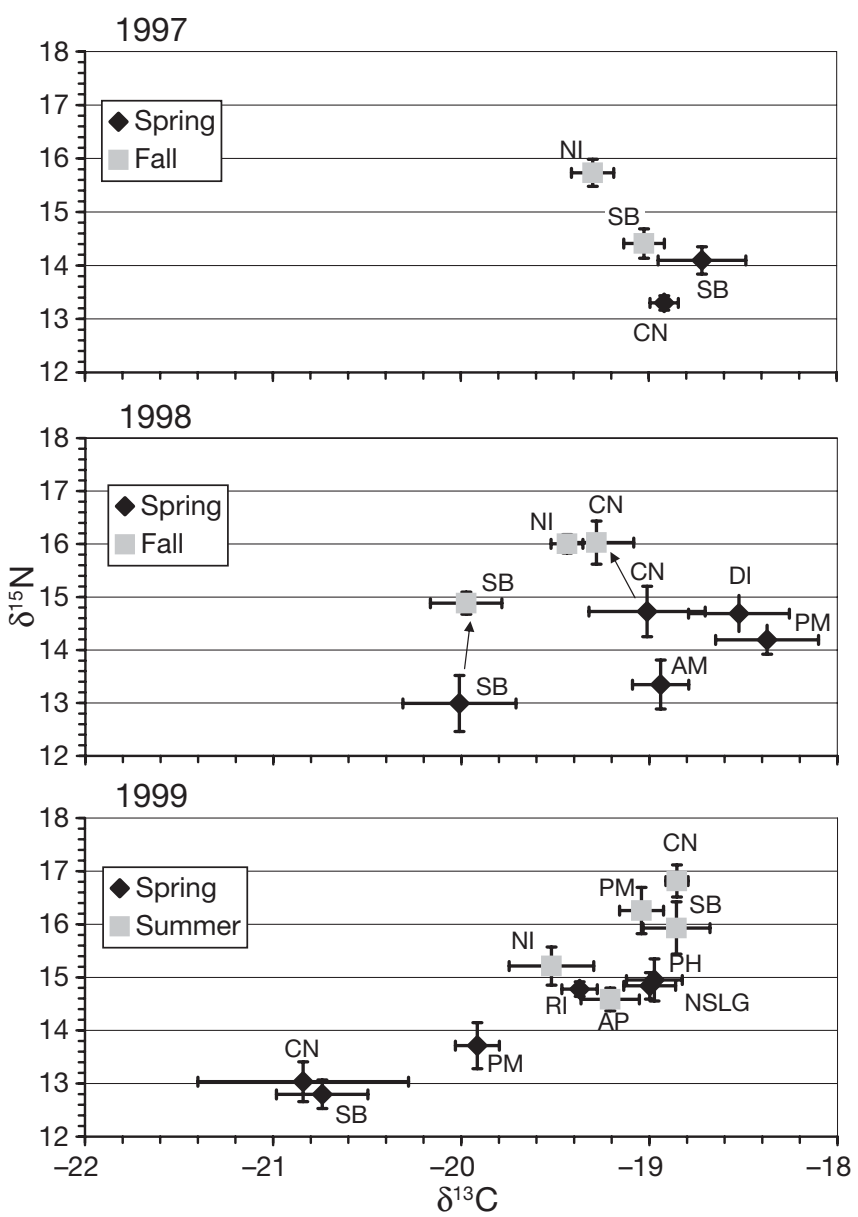

Fig. 7. Puffinus tenuirostris. Shifts in the stable isotope values, $\delta^{15} \mathrm{~N}$ and $\delta^{13} \mathrm{C}$ for short-tailed shearwater tissues (liver) by location, year and season. Sample locations are listed next to their corresponding mean $\pm \mathrm{SE}$. Sample sizes are listed in Table 1. Locations as given in Fig. 1. T.: Thysanoessa

Cape Newenham. These patterns are evident in both the stomach samples and the isotopic data. This finding is surprising because short-tailed shearwaters are highly mobile and form continuous streams of high numbers of individuals that fly great distances (up to 600 to $1124 \mathrm{~km} \mathrm{~d}^{-1}$; Nicholls et al. 1998, Klomp \& Schultz 2000) while chick-rearing and foraging for prey. Thus, we expected that there would be considerable overlap and a lack of significant differences in diet (particularly in stable isotope and fatty acid values) among sampling locations that are on the order of 10 s to 100s of $\mathrm{km}$ apart. This finding also leads us to believe that this spatial and temporal separation occurs on the order of weeks at a time, as evidenced by the separation among locations of isotopic and fatty acid results in tissues that have slower turnover times. Thus, our findings suggest that short-tailed shearwater flocks specialized on particular prey and fed in specific locations for no less than 1 to 2 wk at a time.

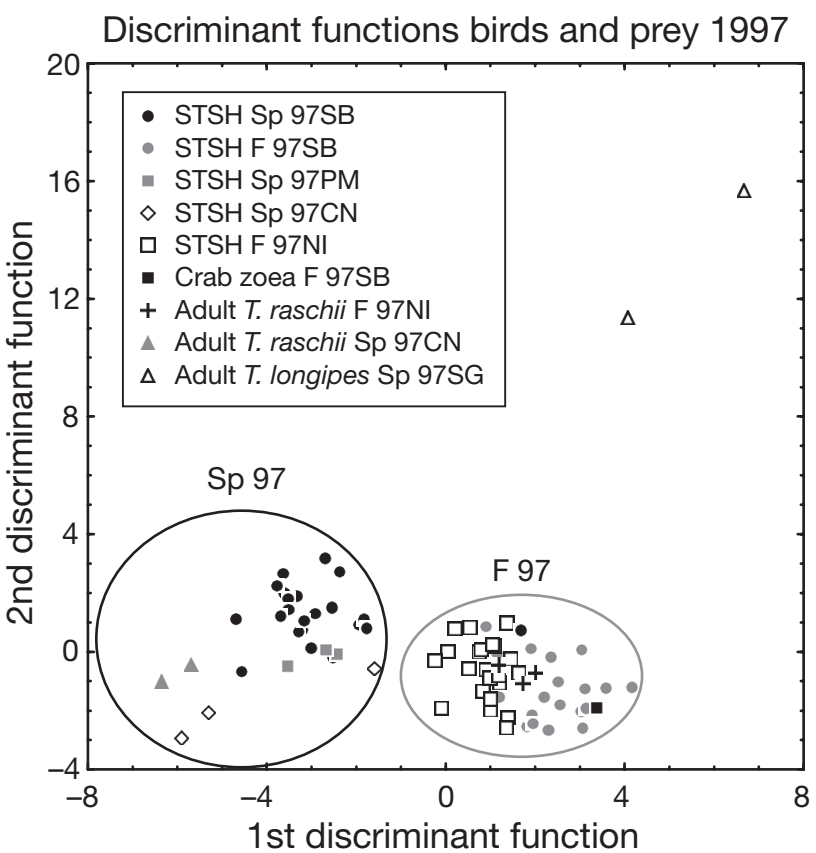

Fig. 8. Puffinus tenuirostris. First 2 discriminant functions describing $94 \%$ of the variation in 17 principal fatty acids sampled from the lipid tissue of short-tailed shearwaters (STSH) and their prey in spring (Sp) and fall (F) 1997, grouped by study location. Sample sizes are listed in Tables $2 \& 3$. Ovals around data represent $95 \%$ confidence intervals between

seasons. Locations as given in Fig. 1. T.: Thysanoessa

We were unable to show whether nutritional stress of shearwaters or elevated $\delta^{15} \mathrm{~N}$ levels in the tissues of prey in fall 1997 and 1998, or elevated isotope levels in the prey, were the cause of elevated $\delta^{15} \mathrm{~N}$ isotope values in birds sampled at Slime Bank and Nunivak Island. Although the analysis of stomach contents suggested that birds were foraging at a lower trophic level in fall (juvenile euphausiids and crab zoea) than in spring when they took adult euphausiids, the stable isotope values in the prey themselves were more elevated in fall compared to spring 1997, possibly because they were feeding on a greater proportion of regenerated production as nitrate was largely depleted from the water column during fall 1997 (Stockwell et al. 2001).

The isotope values we recorded from the liver of shearwaters and their prey in this study are comparable to those found in the pectoralis muscle tissues of short-tailed shearwaters incidentally captured in the large-mesh driftnet (Gould et al. 2000) and gillnet (Minami et al. 1995) fisheries in the North Pacific, despite the difference in diet between this extensive open ocean area and the southeastern Bering Sea. Short-tailed shearwaters that were self-feeding during long foraging trips $\left(-23.8 \% \delta^{13} \mathrm{C}, 8.7 \% \delta^{15} \mathrm{~N}\right)$ and foraging for chicks on short forays $\left(-24.3 \% \delta^{13} \mathrm{C}, 9.2 \%\right.$ $\delta^{15} \mathrm{~N}$ ) in the waters off Tasmania contained lower $\delta^{13} \mathrm{C}$ 


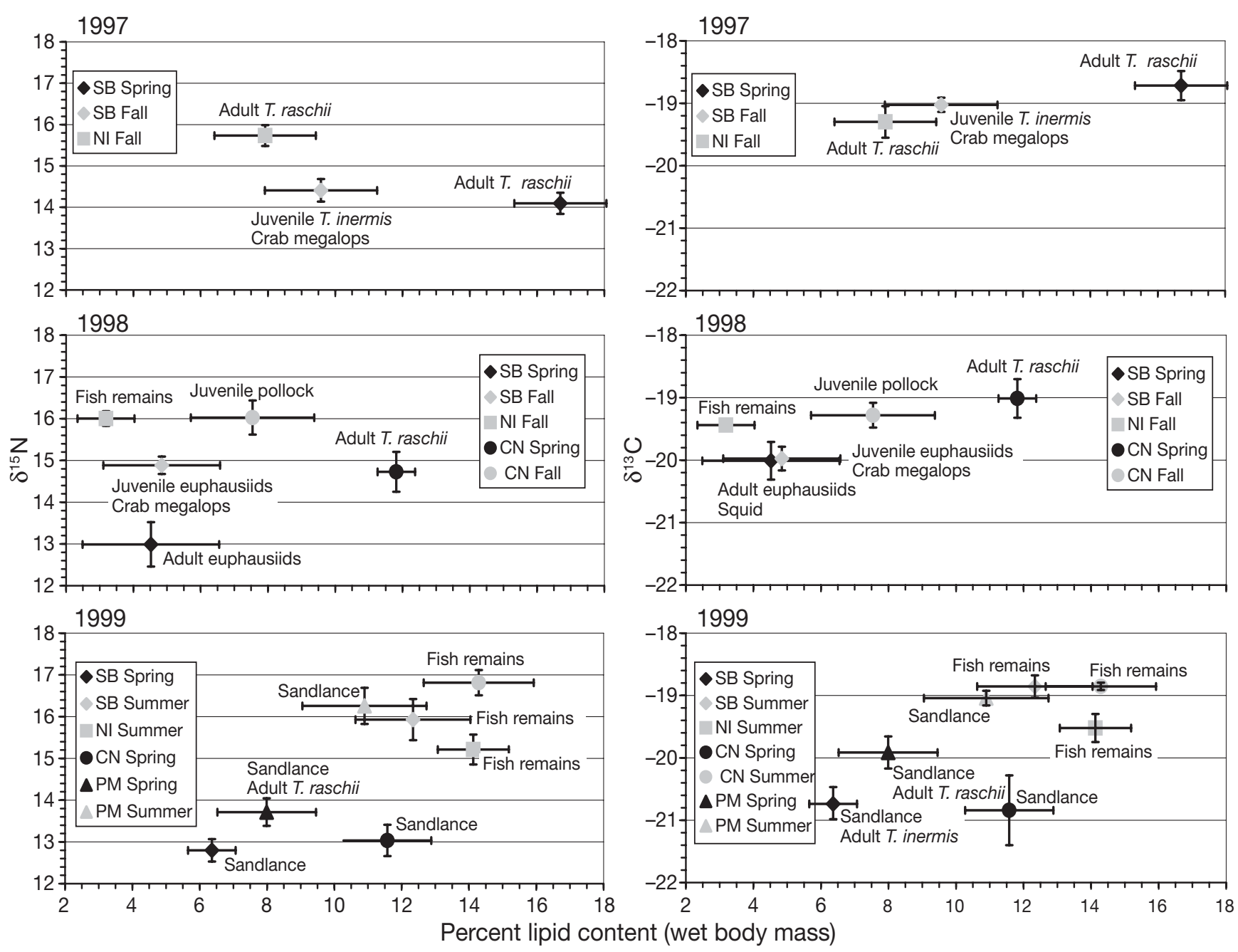

Fig. 9. Puffinus tenuirostris. Stable isotope values, $\delta^{15} \mathrm{~N}$ and $\delta^{13} \mathrm{C}$ for short-tailed shearwater tissues (liver) versus body condition (percent lipid content of wet body mass) among years, seasons, and locations. The primary prey items used are listed next to the mean \pm SE isotope tissue values of birds. Sample sizes are listed in Table 1. Locations as given in Fig. 1. T:: Thysanoessa

and $\delta^{15} \mathrm{~N}$ values in their blood plasma than those recorded in this study (Cherel et al. 2005b). These contrasts could be related to and originate from different isotopic values in the waters of the Antarctic ecosystem compared to that of the eastern Bering Sea, in the prey consumed, and/or differences in tissues sampled.

Our sampling effort of fatty acids from the lipid tissue of short-tailed shearwaters and their prey was not as comprehensive as the diet or isotope sampling and, thus, the results of the fatty acid analysis are more difficult to interpret. However, an important result was that, similar to the diet and isotope results, there significant separation among seasons and locations with respect to fatty acid composition of shearwaters and their prey (Fig. 8). Six of the most common fatty acids found in shearwater tissues and their prey in our study $(14: 0,16: 0,16: 1 n-7,18: 1 n-9,20: 5 n-3,22: 6 n-3)$ also had the greatest percent composition in the stomach oils of short-tailed shearwaters feeding off Tasmania (100\% similarity; Connan et al. 2005).

Short-tailed shearwaters employ a variety of foraging methods that include surface seizing, pursuit plunging with wings and feet, and deep diving from 20 to $70 \mathrm{~m}$ (Skira 1979, Morgan 1982, Morgan \& Ritz 1982, Schneider 1994, Weimerskirch \& Cherel 1998). In our study, we observed short-tailed shearwaters using at least 2 of these methods. When feeding on euphausiid prey, shearwater flocks were large (sometimes greater than 1000 birds flock $^{-1}$ ) and grouped into subunits whereby they leapfrogged among large swarms of euphausiid prey and made short ( $<1 \mathrm{~min})$, shallow dives in pursuit of their prey (C.L. Baduini pers. obs). On occasion, they also seized euphausiids at the surface. This is in contrast to occasions when short-tailed shearwaters 
were observed feeding on fish and flocks were smaller, more dispersed, and had deeper, longer dives ( $>1 \mathrm{~min})$.

The increase in use of prey other than Thysanoessa raschii by short-tailed shearwaters throughout this study may be the result of a decrease in the availability, but not the abundance of $T$. raschii (Hunt et. al 2002a). The presence of a coccolithophore bloom may have reduced the ability of short-tailed shearwaters to find euphausiids from the air in fall 1997 (Baduini et al. 2001a). It is also possible that the summer abundance of T. raschii declined from 1997 to 1999. However, this does not appear to be the case. In the cold year of 1999, acoustically estimated biomass in spring was $8.89 \mathrm{~g} \mathrm{~m}^{-2}$, whereas in the warmer years of 1997 and 1998, their biomass was $1.69 \mathrm{~g} \mathrm{~m}^{-2}$ and $2.44 \mathrm{~g} \mathrm{~m}^{-2}$, respectively (Coyle \& Pinchuk 2002, Table 5). In summer 1999, the numbers of euphausiid eggs and larvae were elevated compared to 1997 and 1998, and Coyle and Pinchuk (2002) suggest that in cold years spawning may be delayed. Thus we would have expected that shearwater diets in summer 1999 would have consisted of a greater proportion of adult euphausiids than in the other years. Lastly, it is possible that shearwater foraging patterns and diet may have varied throughout this study because alternative prey were more abundant or available.

In this study we used 3 methods (1 direct and 2 indirect) to observe spatial and temporal distribution in the transfer of nutrients to short-tailed shearwaters in the southeastern Bering Sea from 1997 to 1999. Each method provided us with evidence that there were significant differences both spatially and temporally in the diet of shearwaters. These results suggest that shearwater flocks form independent foraging units that show surprising cohesion and fidelity to location at a scale of $10 \mathrm{~s}$ to $100 \mathrm{~s}$ of $\mathrm{km}$. If correct, we will need to revise our perception that these birds mix extensively on their foraging grounds and that flocks are constantly coalescing and then breaking up into new associations

Acknowledgements. We thank the captain and crew of the RV 'Alpha Helix' for their support and expertise on research cruises. We also thank the following for their assistance on research cruises and in the laboratory: $\mathrm{M}$. Cahoon, J. Carlson, B. Gibbons, J. Jahncke, N. Karnovsky, J. Lovvorn, B. M. Myers III, M. Ngo, J. Parga, C. Pickens, H. Revilee, G. Savage, and L. Vlietstra. We thank N. Haubenstock and D. Schell for running stable isotope analysis and S. Lang and S. Iverson for running the fatty acid analyses. A. Zaricki assisted with construction of figures. Three anonymous reviewers provided helpful comments that improved the quality of this manuscript. This research was supported by National Science Foundation Office of Polar Programs grant NSFOPP-9617287 to G. L. Hunt Jr. and by the National Oceanic and Atmospheric Administration, Pacific Marine Environmental Laboratory.

\section{LITERATURE CITED}

Ackman RG, Ratnayake WMN, Olsson B (1988) The 'basic' fatty acid composition of Atlantic fish oils: potential similarities useful for enrichment of polyunsaturated fatty acids by urea complexation. J Am Oil Chem Soc 65:136-138

Baduini CL, Hyrenbach KD, Coyle KO, Pinchuk A, Mendenhall V, Hunt GL Jr (2001a) Mass mortality of short-tailed shearwaters in the eastern Bering Sea during summer 1997. Fish Oceanogr 10:117-130

Baduini CL, Lovvorn JR, Hunt GL Jr (2001b) Determining the nutritional condition of short-tailed shearwaters (Puffinus tenuirostris): implications for migratory flight ranges and starvation events. Mar Ecol Prog Ser 222:265-277

Budge SM, Iverson SJ, Bowen WD, Ackman RG (2002) Among-and within-species variability in fatty acid signatures of marine fish and invertebrates on the Scotian Shelf, Georges Bank, and southern Gulf of St. Lawrence. Can J Fish Aquat Sci 59:886-898

Cherel Y, Hobson KA, Bailleul F, Groscolas R (2005a) Nutrition, physiology, and stable isotopes: new information from fasting and molting penguins. Ecology 86:2881-2888

Cherel Y, Hobson KA, Weimerskirch H (2005b) Using stable isotopes to study resource acquisition and allocation in procellariiform seabirds. Oecologia 145:533-540

Connan M, Mayzaud P, Boutoute M, Weimerskirch H, Cherel Y (2005) Lipid composition of stomach oil in a procellariiform seabird, Puffinus tenuirostris: implications for food web studies. Mar Ecol Prog Ser 290:270-290

Coyle KO, Pinchuk AI (2002) The abundance and distribution of euphausiids and zero-age pollock on the inner shelf of the southeast Bering Sea near the Inner Front in 1997-1999. Deep-Sea Res Part II 49:6009-6030

Dahl TM, Falk-Petersen S, Gabrielsen GW, Sargent JR, Hop H, Millar AM (2003) Lipid and stable isotopes in common eider, black-legged kittiwake, and northern fulmar: a trophic study from an Arctic fjord. Mar Ecol Prog Ser 256: $257-269$

Gaston AJ, Nettleship DN (1981) The thick-billed murres of Prince Leopold Island. Can Wildl Serv Monogr Ser 6

Gould PJ, Forsell DJ, Lensink CJ (1982) Pelagic distribution and abundance of seabirds in the Gulf of Alaska and the eastern Bering Sea. US Fish Wildl Serv FWS/OBS-82/48, Washington, DC

Gould PJ, Ostrom P, Walker W (2000) Foods, trophic relationships, and migration of Sooty and short-tailed shearwaters associated with squid and large-mesh driftnet fisheries in the North Pacific Ocean. Waterbirds 23(2):165-186

Guzman J (1981) The wintering of sooty and short-tailed shearwaters (genus Puffinus) in the North Pacific. PhD thesis, University of Calgary

Hobson KA (1990) Stable isotope analysis of marbled murrelets: evidence for freshwater feeding and determination of trophic level. Condor 92:896-903

Hobson KA (1991) Stable isotopic determinations of the trophic relationships of seabirds: preliminary investigations from coastal British Columbia. In: Montevecchi WA, Gaston AJ (eds) Studies of high-latitude seabirds. 1. Behavioral, energetic and oceanographic aspects of seabird feeding ecology. Can Wildl Serv Occas Pap 68: p 16-20

Hobson KA (1993) Trophic relationships among high Arctic seabirds: insights from tissue-dependent stable isotope models. Mar Ecol Prog Ser 95:7-18

Hobson KA, Montevecchi WA (1991) Stable isotopic determination of trophic relationships of great auks. Oceologia 87: 528-531

Hobson KA, Alisauskas RT, Clark RG (1993) Stable-nitrogen 
isotope enrichment in avian tissues due to fasting and nutritional stress: Implications on isotopic analyses of diet. The Condor 95:388-394

Hunt GL Jr, Gould PJ, Forsell DJ, Peterson H Jr (1981) Pelagic distribution of marine birds in the eastern Bering Sea. In: Hood DW, Calder JA (eds) The Eastern Bering Sea: oceanography and resources. National Oceanic and Atmospheric Administration/Bureau of Land Management, Washington, DC, p 689-718

Hunt GL Jr, Baduini CL, Brodeur RD, Coyle KO and 8 others (1999) The Bering Sea in 1998: The second consecutive year of extreme weather-forced anomalies. EOS, Trans Am Geophys Union 80(47):561-566

Hunt GL Jr, Stabeno PJ, Walters G, Sinclair E, Brodeur RD, Napp JM, Bond NA (2002a) Climate change and control of the southeastern Bering Sea pelagic ecosystem. Deep-Sea Res II 49:5821-5853

Hunt GL Jr, Baduini CL, Jahncke J (2002b) Diets of shorttailed shearwaters in the southeastern Bering Sea. Deep-Sea Res II 49:6147-6156

Iverson SJ (1993) Milk secretion in marine mammals in relation to foraging: can milk fatty acids predict diet? Symp Zool Soc Lond No 66, p 263-291

Iverson SJ, Oftedal OT, Bowen WD, Boness DJ, Sampugna J (1995) Prenatal and postnatal transfer of fatty acids from mother to pup in the hooded seal (Cystophora cristata). J Comp Physiol B 165:1-12

Iverson SJ, Frost KJ, Lowry LF (1997) Fatty acid signatures reveal fine scale structure of foraging distribution of harbor seals and their prey in Prince William Sound, Alaska. Mar Ecol Prog Ser 151:255-271

Iverson SJ, Frost KJ, Lang SLC (2002) Fat content and fatty acid composition of forage fish and invertebrates in Prince William Sound, Alaska: Factors contributing to among and within species variability. Mar Ecol Prog Ser 241:161-181

Iverson SJ, Field C, Bowen WD, Blanchard W (2004) Quantitative fatty acid signature analysis: a new method of estimating predator diets. Ecol Monogr 74(2):211-235

Jahncke J, Coyle KO, Zeeman SI, Kachel NB, Hunt GL Jr (2005) Distribution of foraging shearwaters relative to inner front of southeastern Bering Sea. Mar Ecol Prog Ser 305:219-233

Klomp NI, Schultz MA (2000) Short-tailed shearwaters breeding in Australia forage in Antarctic waters. Mar Ecol Prog Ser 194:307-310

McConnaughey T, McRoy CP (1979) Food-web structure and the fractionation of carbon isotopes in the Bering Sea. Mar Biol 53:257-262

Michener RH, Schell DM (1994) Stable isotope ratios as tracers in marine aquatic food webs. In: Latjhand $\mathrm{K}$, Michener $\mathrm{R}$ (eds) Stable isotopes in ecology and environmental science. Blackwell Scientific, Oxford, p 138-157

Minami H, Minagawa M, Ogi H (1995) Changes in stable carbon and nitrogen isotope ratios in sooty and short-tailed shearwaters during their northward migration. Condor 97: $565-574$

Editorial responsibility: Howard I. Browman (Associate Editor-in-Chief), Storebø, Norway
Morgan WL (1982) Feeding methods of the short-tailed shearwater Puffinus tenuirostris. Emu 82:226-227

Morgan WL, Ritz DA (1982) Comparison of the feeding apparatus in the muttonbird Puffinus tenuirostris (Temmink) and the fairy prion Pachyptila turtur (Kuhl) in relation to the capture of krill, Nyctiphanes austalis (Sars). J Exp Mar Biol Ecol 59:61-75

Napp JM, Hunt GL Jr (2001) Anomalous conditions in the southeastern Bering Sea, 1997: Linkages among climate, weather, ocean and biology. Fish Oceanogr 10:61-68

Napp JM, Baier CT, Brodeur RD, Coyle KO, Shiga N, Mier K (2002) Interannual and decadal variability in zooplankton communities of the southeast Bering Sea shelf. Deep-Sea Res II 49:5991-6008

Nicholls DG, Stampton P, Klomp NI, Schultz M (1998) Postbreeding flight to Antarctic waters by a short-tailed shearwater Puffinus tenuirostris. Emu 98:79-82

Pinkas L, Oliphant MS, Iverson ILK (1971) Food habits of albacore, bluefin tuna, and bonito in California waters. Calif Fish Game Fish Bull 152, State of California, Department of Fish and Game, Sacramento, CA

Raclot T, Groscolas R, Cherel Y (1998) Fatty acid evidence for the importance of myctophid fishes in the diet of king penguins, Aptenodytes patagonicus. Mar Biol 132: 523-533

Rau GH, Ainley DG, Bengston JL, Torres JJ, Hopkins TL (1992) ${ }^{15} \mathrm{~N} /{ }^{14} \mathrm{~N}$ and ${ }^{13} \mathrm{C} /{ }^{12} \mathrm{C}$ in Weddell Sea birds, seals, and fish: implications for diet and trophic structure. Mar Ecol Prog Ser 84:1-8

Schneider DC (1994) Scale-dependent spatial dynamics: Marine birds in the Bering Sea. Biol Rev 68:579-598

Schneider DC, Hunt GL Jr (1982) Carbon flux to seabirds in water with different mixing regimes in the southeastern Bering Sea. Mar Biol 67:237-344

Schneider DC, Shuntov VP (1993) The trophic organization of the marine bird community in the Bering Sea. Rev Fish Sci 1:311-335

Schneider DC, Hunt GL Jr, Harrison NM (1986) Mass and energy transfer to seabirds in the southeastern Bering Sea. Cont Shelf Res 5(1/2):241-257

Skira IJ (1979) Underwater feeding by short-tailed shearwaters. Emu 79:43

Stabeno PJ, Hunt GL Jr (2002) Overview of the Inner Front and Southeast Bering Sea carrying capacity programs. Deep-Sea Res II 49:6157-6168

Stockwell DA, Whitledge TE, Zeeman S, Coyle KO, Napp JM, Brodeur RD, Pinchuk AI, Hunt GL Jr (2001) Aomalous conditions in the south-eastern Bering Sea, 1997: nutrients, phytoplankton and zooplankton. Fish Oceanog 10: 99-116

Weimerskirch H, Cherel Y (1998) Feeding ecology of shorttailed shearwaters: breeding in Tasmania and foraging in the Antarctic? Mar Ecol Prog Ser 167:261-274

Wilson RP, La Cock GD, Wilson MP, Mollagee F (1985) Differential digestion of fish and squid in jackass penguins, Spheniscus demersus. Ornis Scandinavica 16:77-79

Submitted: November 8, 2005; Accepted: January 17, 2006 Proofs received from author(s): July 26, 2006 